\title{
Improved Cognition and Preserved Hippocampal Fractional Anisotropy in Subjects Undergoing Carotid Endarterectomy
}

\section{Adam Bernstein}

University of Arizona https://orcid.org/0000-0002-1770-6603

Gloria Guzman

University of Arizona

Juan Arias

University of Arizona

Denise Bruck

University of Arizona

\section{Scott Berman}

University of Arizona Medical Center - University Campus: Banner University Medical Center Tuscon

Luis Leon

University of Arizona

John Pacanowski

University of Arizona

Wei Zhou

University of Arizona

Kay Goshima

University of Arizona

Tze-Woei Tan

University of Arizona

Maria Altbach

University of Arizona

Theodore Trouard

University of Arizona

Craig Weinkauf ( $\square$ ccweinkauf@email.arizona.edu )

University of Arizona

\section{Research Article}

Keywords: Carotid Artery Atherosclerosis, Diffusion Magnetic Resonance Imaging, Fractional Anisotropy, Neurocognitive Assessment 
Posted Date: February 11th, 2021

DOI: https://doi.org/10.21203/rs.3.rs-185232/v1

License: (c) (i) This work is licensed under a Creative Commons Attribution 4.0 International License. Read Full License 


\section{Abstract}

A growing body data indicates that extracranial carotid artery disease can contribute to cognitive impairment. However, there have been mixed reports regarding the benefit of carotid endarterectomy (CEA) as it relates to preserving cognitive function. In this work, diffusion magnetic resonance imaging (dMRI) is used to provide insight into structural brain changes that occur because of significant carotid artery stenosis, as well as changes that occur in response to CEA. The study design was a prospective, non-randomized, controlled study that enrolled patients with asymptomatic carotid stenosis. Fourteen subjects had severe ECAD $\geq 70 \%$ stenosis in at least one carotid artery and were scheduled to undergo surgery). Thirteen had ECAD with $<70 \%$ stenosis or occluded, therefore not requiring surgery. All subjects underwent neurocognitive testing using the Montreal Cognitive Assessment test and high angular resolution, multi-shell diffusion magnetic resonance imaging (dMRI) of the brain at baseline and at foursix months follow-up. Changes in MoCA scores as well as in Fractional anisotropy along the hippocampus were compared at baseline and follow-up. At baseline, fractional anisotropy (FA) was significantly lower along the ipsilateral hippocampus in subjects with severe ECAD compared to subjects without severe ECAD. MoCA scores were lower in these individuals but this did not reach statistical significance. At follow-up, MoCA scores increased significantly in subjects who underwent CEA and remained statistically equal in control subjects that did not have CEA. FA remained unchanged in the CEA group, and decreased in the control group. This study suggests that CEA improves cognition and preserves hippocampal white matter structure compared to control subjects not undergoing CEA.

\section{Background}

Extracranial carotid artery disease (ECAD) is associated with an increased risk of stroke (Flaherty et al., 2013). Growing evidence has also shown that people with ECAD are at increased risk of developing cognitive impairment (Lal et al., 2017). In fact, it has been demonstrated that vascular disease, in general, increases the risk of developing any form of dementia (Bunch et al., 2020; Lal et al., 2017; Suemoto et al., 2011; Wendell, Zonderman, Metter, Najjar, \& Waldstein, 2009). Despite this, guidelines for the treatment of ECAD are focused only on stroke prevention (Ricotta et al., 2011). This is in part because recent studies evaluating the effect of carotid revascularization procedures have produced mixed results on their effect on cognition.

Several studies have investigated the effects of carotid interventions on cognition, including carotid artery stenting and carotid endarterectomy (CEA), with variable results. Some studies have demonstrated improvements in cognition following carotid revascularization procedures (Dempsey et al., 2018; Kojima et al., 2016; Nakahara et al., 2020). However, others have found no measurable benefit of CEA or stenting (De Rango et al., 2008; Heller \& Hines, 2017; Rothwell \& Goldstein, 2004). An asymptomatic carotid surgery trial found that there was no significant reduction in risk of dementia between patients who received immediate versus delayed CEA assessed at 15 years following CEA (Rothwell \& Goldstein, 2004). Another review of 32 studies assessing cognition following carotid artery stenting or CEA also concluded that there was no evidence that either procedure had an effect on neuropsychological test results, noting 
that there was a lack of appropriately powered studies that would be necessary to draw more concrete conclusions (De Rango et al., 2008). A more recent review by Heller and Hines found similar results (Heller \& Hines, 2017). In addition to other confounding factors and variability in study techniques and study populations, it is possible that there are mixed and often inconclusive results due to the lack of robust clinical tools for assessing cognition and brain health.

While neuropsychological testing is one of the few tools available to assess cognition, neuroimaging techniques, specifically magnetic resonance imaging (MRI), provide insight into the organization and structure of the brain. Such imaging may provide valuable information about changes in the brain as a result of intervention in patients with significant carotid disease.

Diffusion magnetic resonance imaging (dMRI) is a noninvasive MRI technique that uses the motion of water at a microscopic scale to probe the local tissue microstructure (Jones et al., 2002). Diffusion tensor imaging (DTI), a dMRI technique that quantifies water mobility, and infers tissue structure and organization through measures such as fractional anisotropy (FA), has been used to investigate the association between diffusion parameters and cognitive impairment, including Alzheimer's disease (Anderson, Litvack, \& Kaye, 2005; Bartzokis, 2004; Borroni et al., 2007; Bozzali, 2002; Zhang et al., 2009). FA is a widely utilized metric of tissue microstructure, which describes the relative preference of water to diffuse along one direction compared to any other direction (e.g. along an axon in white matter). FA is highly sensitive to changes in white matter structure at a microscopic scale, such as demyelination, atrophy, edema, etc., and can be used to infer changes in white matter structure in certain disease states. In addition to probing the microscopic structure of tissues, dMRI has been used to map out the macroscopic organization of the brain using tractography.

Advanced dMRI techniques that more completely characterize the microscopic organization of tissue, such as high angular resolution diffusion imaging (HARDI) and constrained spherical deconvolution (CSD), have also been used to characterize brain structure in cognitive impairment and dementia (Mito et al., 2018), demonstrating significant decreases in fiber density throughout the brain. Additionally, techniques such as HARDI and CSD, which can capture the complex organization of neuronal fibers, are well suited to diffusion tractography, which can provide valuable macroscopic structural information about the brain (Tournier, Calamante, \& Connelly, 2012), These techniques have also recently been used to show changes in brain structure associated with cognition (Avirame et al., 2015; Lin, Tu, Chern, Hsiao, \& Chang, 2014), DTI and CSD can be combined to provide a combination of macroscopic and microscopic structural information. In this work we utilized CSD to provide macroscopic information about the organization of major white matter pathways throughout the brain using tractography, and we used DTI to measure the microstructure of white matter along such major white matter pathways. This technique, a hybrid of techniques such as voxel-based analysis, tract-based spatial statistics (Smith et al., 2006), and region of interest-based analysis, avoids the noisy statistics and registration errors of a voxelbased technique (IIfanoglu et al., 2016), and the averaging effect of a large ROI-based analysis by finding local means of diffusion parameters along the length of white matter structures of interest. 
The goal of this work was to investigate the effects of CEA on subjects with significant carotid artery stenosis, particularly on preserving or restoring cognitive function and brain connectivity. A combination of neuropsychological testing and advanced neuroimaging techniques were used to provide a deeper insight into the structure of the brain before and after intervention in this study.

\section{Methods}

\subsection{Study design and subject recruitment}

This study was a prospective, non-randomized controlled study that enrolled 27 subjects with asymptomatic extracranial carotid artery stenosis over 1.5 years. Asymptomatic carotid disease is defined as having no stroke or TIA in the previous 6 months, as evaluated by their vascular surgeon. All research involving human subjects was approved by the Institutional Review Board (IRB) of the University of Arizona. Written and oral informed consents were obtained from all subjects. Fourteen surgical subjects with severe unilateral carotid artery disease, defined as 70-99\% stenosis by ultrasound (peak systolic velocity $>230 \mathrm{~cm} / \mathrm{sec}$ and ICA $/$ CCA ratio $>4$ or end diastolic velocity $>100 \mathrm{~cm} / \mathrm{sec}$ ) of the internal carotid artery on doppler ultrasound that were scheduled for CEA were enrolled in the study. Thirteen subjects with carotid artery disease not classified as severe ( $<70 \%$ stenosis) or occlusion in at least one carotid artery were enrolled in the study as controls and did not undergo CEA. For all patients, inclusion criteria were: 50-80 years of age, English-speaking, able to give consent, atherosclerosis seen by ultrasound in at least one carotid artery and at least 2 cardiovascular risks including hypertension, hyperlipidemia, diabetes or history of smoking as defining in clinical records, and ability to abstain from caffeine for 4 hours. Exclusion criteria: Depression (>12 on Geriatric Depression Scale), dementia ( $<18$ on MoCA), pregnant, inability to give consent, prisoner, contraindication to MRI, neurological disorder, history of loss of consciousness with sequelae, planning to move out of area within 1 year, end-stage renal disease, terminal cancer, drug/alcohol abuse.

\subsection{Carotid stenosis calculation}

Although study enrollment was based on duplex ultrasound criteria, all subjects had high resolution MRI of the carotid arteries for uniform calculation carotid stenosis evaluation using NASCET criteria. This evaluation resulted in similar or decreased percent stenosis for subjects compared to ultrasound evaluation. MRI-derived percent stenosis is reported in Table 1.

\subsection{Neurocognitive Testing}

All subjects underwent neurocognitive testing in the form of the Montreal Cognitive Assessment (MoCA) at baseline, and at 4-6 month follow-up. Subjects with severe ECAD completed their baseline MoCA within 1-week prior to undergoing CEA. For comparison of MoCA between controls and subjects undergoing CEA at baseline, a student's t-test was used. For comparison between baseline and follow-up, a paired t-test was used to compare differences in MoCA scores over time. Significance was set at $p<0.05$. 


\subsection{Image Data Acquisition}

All imaging data was collected on a 3T Skyra MRI scanner (Siemens Medical Solutions, Erlangen, Germany, VE 11A), using the body RF coil for excitation and a 32-channel phased array head coil for reception. A 3D T -weighted image (MPRAGE parameters: TR=2530ms, TE=3.3ms, Tl=1000ms, resolution=1mm $\times 1 \mathrm{~mm} \times 1 \mathrm{~mm}$, matrix=256 $\times 256$ ) was acquired for anatomical reference. The dMRI experiment employed 2D spin-echo single-shot echo-planar imaging (EPI) with $69 \times 2 \mathrm{~mm}$ slices (no gap); a multiband factor of $3 ; \mathrm{TR} / \mathrm{TE}=3700 / 115 \mathrm{~ms}$; matrix size $=128 \times 128 ; \mathrm{FOV}=256 \times 256 \mathrm{~mm}$, and inplane GRAPPA factor of 2. Diffusion weighting included $14 \mathrm{~b}=0 \mathrm{~s} / \mathrm{mm}^{2}, 20 \mathrm{~b}=1000 \mathrm{~s} / \mathrm{mm}^{2}, 32 \mathrm{~b}=$ $2000 \mathrm{~s} / \mathrm{mm}^{2}$, and $64 \mathrm{~b}=3000 \mathrm{~s} / \mathrm{mm}^{2}$, distributed around a sphere using a multi-shell electrostatic repulsion scheme similar to that described by Caruyer et al. (Caruyer, Lenglet, Sapiro, \& Deriche, 2013), producing a direction set that is evenly distributed on each shell and non-collinear between shells. In addition, a single $b=0 \mathrm{~s} / \mathrm{mm}^{2}$ was collected with reversed phase encoding for use in EPI distortion correction.

\subsection{Image Processing and Analysis}

Processing and analysis for dMRI data are depicted graphically in Figure 1. Original dMRI images went through several pre-processing steps. Gibbs ringing correction was performed on the dMRI images using the method described by Veraart et al. (Veraart, Fieremans, Jelescu, Knoll, \& Novikov, 2016). EPI distortion correction was then performed using FSL's TOPUP algorithm using all $\mathrm{b}=0 \mathrm{~s} / \mathrm{mm}^{2}$ images collected (Andersson, Skare, \& Ashburner, 2003). Eddy current and motion correction was then performed using FSL's eddy algorithm (Andersson \& Sotiropoulos, 2016), and the diffusion encoding directions were rotated to adjust for head motion (Leemans \& Jones, 2009). Next, local principal component analysis (LPCA) was used to denoise the dataset (Manjón et al., 2013). Bias field correction, as implemented as a part of the ANTS software package, was performed as previously described by Tustison et al. (Tustison et al., 2010). Finally, the image data were left-right flipped for subjects undergoing surgery on the right hand side so that group and template analysis could be readily performed on ipsilateral (left) and contralateral (right) sides relative to surgery.

Diffusion tensor fitting for each subject was carried out using weighted linear least squares fitting, and FA maps were estimated from the derived eigenvalues using in-house Python code (Basser, Mattiello, \& LeBihan, 1994). A group average template was then created using the constrained spherical deconvolution (CSD) (Tournier, Calamante, \& Connelly, 2007) based fiber orientation distribution registration in MRtrix (Raffelt et al., 2011) Multi-shell, multi-tissue CSD was performed on each subject's dMRI data using a group-averaged response function (Jeurissen, Tournier, Dhollander, Connelly, \& Sijbers, 2014), and each subject's resulting fiber orientation distribution function (fODF) maps were used to create a group fODF template. The image warps used to register individual subject fODFs to the group template fODF image were then used to warp each subjects FA map onto a generated FA template. Group averaged FA along specific white matter tracts were calculated as described below. 
White matter tractograms were generated for 18 major white matter pathways as depicted in Figure 2. These were defined using regions of interest drawn on the fODF template created above, similar to those described by Wakana et al.(Wakana et al., 2007) These tractograms were then resampled at 100 equidistant points along their length using MRtrix. Finally, FA was sampled along the length of each track using the FA maps registered to the template image described above. FA was computed along the tracks at all 100 locations for each subject, and group-wise statistics were performed using the average alongtrack measures. For comparison of FA between controls and subjects undergoing CEA at baseline, a 2way t-test was used. For comparison between baseline and follow-up imaging sessions, a paired t-test was used to compare differences in FA over time. Significance was set at $p<0.05$.

\section{Results}

As seen in Table 1, subjects in both arms of the study are similar in age, gender, cardiovascular risks and education levels. The only difference is that the control subjects not undergoing carotid endarterectomy have significantly decreased percent carotid stenosis. No subjects in either arm of the study had cerebrovascular events during the course of the study.

\section{Table 1: Subject Demographics}




\section{Control \\ $(\mathrm{N}=13)$}

Age

Mean (SD)

Median [Min, Max]

Highest Stenotic Carotid Artery (\%)

Mean (SD)

Median [Min, Max]

Gender

Female

Male

\section{Smoking}

Current

Former

Never

\section{Diabetes Mellitus}

No

Yes

\section{Hyperlipidemia}

No

Yes

\section{Hypertension}

No

Yes

\section{Education}

Grade School

High School

Some College

\section{Aspirin}

No
68.8 (8.62)

$65.0[56.0,80.0]$
Surgery

$(\mathrm{N}=13)$
P-value

$69.9(8.06)$

1

$71.0[53.0,80.0]$
26.1 (37.8)

$0[0,100]$

$4(30.8 \%)$

9 (69.2\%)

$2(15.4 \%)$

$10(76.9 \%)$

$1(7.7 \%)$

$10(76.9 \%)$

3 (23.1\%)

$12(92.3 \%)$

$1(7.7 \%)$
1

10 (76.9\%)

3 (23.1\%)

0.313

$6(46.2 \%)$

4 (30.8\%
3 (23.1\%)

10 (76.9\%)
2 (15.4\%)

11 (84.6\%)
2 (15.4\%)

11 (84.6\%)
$6(46.2 \%)$

7 (53.8\%)
5 (38.5\%)

2 (15.4\%)

$6(46.2 \%)$

$4(30.8 \%)$

Page 8/21
3 (23.1\%)

0.545
4 (30.8\%)

$6(46.2 \%)$ 
Yes

\section{Statin}

No

Yes

Derived from Wilcoxon rank-sum test for continuous variables and Fisher's exact test for categorical variables

MoCA test scores at baseline and 4-6 month follow-up for subjects that underwent CEA (CEA group) and subjects that had no intervention (control group) are shown in Figure 3. At baseline, MoCA scores were lower in the CEA group (those with greater carotid stenosis) than in the control group (24.23 \pm 2.55 vs. $25.85 \pm 2.41, P=0.11$ ), but this difference was not statistically significant. There was a significant increase in MoCA scores in the CEA group from baseline to follow-up (24.23 \pm 2.55 vs. $25.92 \pm 2.25, P=$ 0.02) while there was no significant difference in MoCA scores in the control group between baseline and follow-up $(25.85 \pm 2.41$ vs. $26.54 \pm 1.33, P=.11)$. The changes seen in overall MoCA score between baseline and follow-up testing in the CEA group were driven primarily by changes in the visuospatial/executive cognitive domain $(P=0.02)$ and the abstraction domain $(P=0.04)$, as displayed in the sub-panels in figure 3. Other cognitive domains in the MoCA (orientation, naming, attention, language) were statistically equal between baseline and follow-up scores.

Comparison of FA along all 18 defined white matter pathways between the treatment and control group at baseline demonstrated statistically significant differences within the hippocampus ipsilateral to the affected carotid artery in the treatment group. There were no statistically significant differences along other white matter pathways analyzed. Plots of FA along the length of the hippocampal part of the cingulum bundle at baseline in control and CEA groups on the affected side of the brain are shown in Figure 4A. For both groups, FA values are low towards the anterior part of the tract, increase towards the middle and dip before moving higher towards the posterior end of the tract. There were significantly lower values of FA in the posterior portion of the hippocampus on the affected side of the CEA group (red line in Figure 4A). The average differences in FA are plotted along the hippocampus in Figure 4B for anatomical reference where the light blue color indicates the location where there are statistically lower values of FA in the CEA group.

A similar comparison was performed to explore changes in FA along the hippocampus in the treatment group and the control group between baseline and 4-6 month follow-up. Figure 5A plots the average FA along the hippocampus at baseline (blue) and follow-up (light blue) in the control group. Figure 5B plots the FA along the hippocampus in the CEA group at baseline (red) and follow-up (light red) and showed no significant changes in FA. In contrast to the treatment group, the control group showed significant decreases in FA along the posterior portion of the hippocampus. The region of decrease is roughly the same region where there were significant differences in FA between CEA and control groups were seen at baseline. As above, figure $5 \mathrm{C}$ and $5 \mathrm{D}$ plot the average change in $\mathrm{FA}$ along the length of the hippocampus 
for the control group and the CEA group respectively. There were no significant changes in FA along any of the other white matter pathways in either group.

\section{Discussion}

In this work, effects of extracranial carotid artery disease and carotid endarterectomy on brain structure and cognitive function were evaluated in asymptomatic subjects with severe carotid stenosis $(70-99 \%)$ who underwent CEA and control subjects with $<70 \%$ carotid stenosis or occluded who did not undergo CEA. A combination of neurocognitive testing and neuroimaging was used to provide unique insight into brain changes in this population. Our study utilized state-of-the-art dMRI techniques which have not previously been used to evaluate this patient population in order to maximize spatial sensitivity to subtle changes in specific regions of the brain.

Baseline comparison between groups of control subjects and those with severe ECAD suggests those with severe disease have worse cognitive function, yet this did not reach statistical significance. This finding is consistent with the findings presented by Lal et al. (Lal et al., 2017), who found that ECAD is associated with worse cognitive function. It is likely that our study was underpowered to reach statistical significance and our control population was not optimized to answer whether ECAD contributes to cognition or brain changes as control subjects have carotid artery disease in order to optimize controlling for effects of carotid endarterectomy. However, we did find significant differences in microstructure of the brain at baseline, as measured by FA along the posterior portion of the hippocampus. These differences are statistically significant on the side affected by the severe carotid stenosis and are consistent with our hypothesis that ECAD is associated with white matter alterations, particularly in the hippocampus, a structure with a well-documented role in the progression of dementia. Further, other work has documented a relationship between the extent of ECAD and decreased hippocampal volume (Baradaran et al., 2020), consistent with our findings of hippocampal involvement. In this cohort of asymptomatic subjects, such changes in white matter could potentially be early markers of underlying disease not necessarily associated with stroke.

There are limited studies documenting the effect of CEA or carotid artery stenting (CAS) on brain structure and function using dMRI. The data presented here suggest that CEA may have a protective effect on white matter as well as cognition. In the CEA intervention group, FA showed no significant decrease over time, while the control group had a significant decrease in FA over 4-6 months. It may be that CEA prevents reductions in FA in the hippocampal portion of the cingulum bundle. This result is consistent with the findings of Porcu et al (Porcu et al., 2019), who found increases in cognitive performance and white matter connectivity following CEA.

The data in this work suggest that, in addition to preventing stroke, CEA may have a protective effect in preserving white matter and cognition, and that ECAD may pose additional risks for cerebral injury and degeneration, even in the absence of stroke. However, the study has limitations. The key limitation of this study is the low number of subjects, making it prone to type I and type II statistical errors. As with other 
studies on this topic (De Rango et al., 2008; Dempsey et al., 2018; Kojima et al., 2016; Nakahara et al., 2020), there are relatively few subjects and drawing clinically significant conclusions is not possible. Another limitation is that subjects undergoing CEA are not randomized. To account for this, a control population with similar cardiovascular risks and significant carotid stenosis (including subjects with carotid occlusion) was used to mitigate potential confounding variables for evaluation of interventions. Related to this, our control group has slightly decreased carotid stenosis compared to the intervention group; this is necessary because asymptomatic subjects with greater stenosis would be undergoing surgery in the clinical practices that contributed to the study. Alternatively, if we recruited subjects with $\geq 70 \%$ stenosis who were not undergoing surgery, it would likely introduce more variation into the cohorts because only subjects with severe comorbidities and/or short life expectancies would not be offered surgical intervention. The 4-6-month follow-up used in this study may be another limitation because brain changes in white matter and cognition can occur over years, yet other studies have described changes in similar parameters over similar time periods (Cavallari et al., 2017; Power et al., 2019). However, given our results, we are encouraged that following similar populations over extended periods may provide evidence that CEA has protective benefits in this population. Overall, this field of work is limited because there are numerous neurocognitive tests available to assess cognition, but there is still no ideal, consistent, reproducible test that accurately quantifies the extent of cognitive decline (Spencer et al., 2013). This further highlights the need to pursue more rigorous and reproducible measures, such as the imaging techniques utilized here.

In conclusion, this work demonstrated that in a small cohort of subjects, CEA prevented significant changes in white matter fractional anisotropy and led to an increase in MoCA scores. These data suggest that more quantitative measures, such as fractional anisotropy from diffusion-weighted imaging, may provide insight into the effects of ECAD and CEA on the brain.

\section{Declarations}

\section{Acknowledgements}

This work was funded by the Arizona health sciences center translational imaging program project stimulus at the University of Arizona, University of Arizona Health Sciences Career Development Award, Arizona Alzheimer's Association, and private research funding from Dick and Jan Highberger.

Funding: This work was funded by the Arizona health sciences center Translational Imaging Program Project Stimulus (TIPPS) at the University of Arizona, University of Arizona Health Sciences Career Development Award, Arizona Alzheimer's Association, and private research funding from Dick and Jan Highberger. No funding source had input into study design, data analysis or plan to publish.

Conflicts of Interest: None of the authors declares that they have conflicts of interest.

Ethics Approval: This study was conducted in accordance with the Delcaration of Helsinki, and was approved by the institutional review board at the University of Arizona. 
Consent to Participate: All subjects signed written informed consent.

Consent for Publication: All authors listed on this manuscript are in agreement with the findings and their interpretation and provide consent for publication of the this manuscript.

Availability of data and material: Data will not be made available for patient privacy.

Code Availability: All analysis software is publicly available as described in the methods section of this manuscript.

Author's Contributions: Adam Bernstein conducted the majority of data analysis and statistical analysis, composed the imaging protocol, and wrote the majority of the manuscript presented. Gloria Guzman provided expert consultation as a board certified radiologist, helped with problem formulation and data analysis strategies. Juan Arias conducted statistical analysis and interpretation of neurocognitive testing data. Denise Bruck conducted neurocognitive testing and data acquisition. Scott Berman, Luis Leon, John Pancanowski, Wei Zhou, Kay Goshima, Tze-Woei Tan, and Zachary Taylor all performed carotid endarterectomies on enrolled subjects and provided clinical correlations and commentary on the results. Maria Altbach and Theodore Trouard played a key role in the problem formulation, experimental design, funding acquisition, data collection and composition of the manuscript. Craig Weinkauf was the primary investigator of this work, formulated the primary research question, developed the overall experimental design and analysis strategy, made significant contributions to the writing of the manuscript and data analysis, and provided mentorship throughout the project.

\section{References}

Anderson, V. C., Litvack, Z. N., \& Kaye, J. A. (2005). Magnetic Resonance Approaches to Brain Aging and Alzheimer Disease-associated Neuropathology. Topics in Magnetic Resonance Imaging, 16(6), 439-452. https://doi.org/10.1097/01.rmr.0000245458.05654.d0

Andersson, J. L. R., Skare, S., \& Ashburner, J. (2003). How to correct susceptibility distortions in spin-echo echo-planar images: Application to diffusion tensor imaging. Neurolmage, 20(2), 870-888. https://doi.org/10.1016/S1053-8119(03)00336-7

Andersson, J. L. R., \& Sotiropoulos, S. N. (2016). An integrated approach to correction for off-resonance effects and subject movement in diffusion MR imaging. Neurolmage. https://doi.org/10.1016/j.neuroimage.2015.10.019

Avirame, K., Lesemann, A., List, J., Witte, A. V., Schreiber, S. J., \& Flöel, A. (2015). Cerebral autoregulation and brain networks in occlusive processes of the internal carotid artery. Journal of Cerebral Blood Flow and Metabolism: Official Journal of the International Society of Cerebral Blood Flow and Metabolism, 35(2), 240-247. https://doi.org/10.1038/jcbfm.2014.190 
Baradaran, H., Demissie, S., Himali, J. J., Beiser, A., Gupta, A., Polak, J. F., ... Romero, J. R. (2020). The progression of carotid atherosclerosis and imaging markers of dementia. Alzheimer's \& Dementia:

Translational Research \& Clinical Interventions, 6(1). https://doi.org/10.1002/trc2.12015

Bartzokis, G. (2004). Age-related myelin breakdown: a developmental model of cognitive decline and Alzheimer's disease. Neurobiology of Aging, 25(1), 5-18.

https://doi.org/10.1016/j.neurobiolaging.2003.03.001

Basser, P. J., Mattiello, J., \& LeBihan, D. (1994). MR diffusion tensor spectroscopy and imaging. Biophysical Journal, 66(1), 259-267. https://doi.org/10.1016/S0006-3495(94)80775-1

Borroni, B., Brambati, S. M., Agosti, C., Gipponi, S., Bellelli, G., Gasparotti, R., ... Padovani, A. (2007). Evidence of White Matter Changes on Diffusion Tensor Imaging in Frontotemporal Dementia. Archives of Neurology, 64(2), 246. https://doi.org/10.1001/archneur.64.2.246

Bozzali, M. (2002). White matter damage in Alzheimer's disease assessed in vivo using diffusion tensor magnetic resonance imaging. Journal of Neurology, Neurosurgery \& Psychiatry, 72(6), 742-746. https://doi.org/10.1136/jnnp.72.6.742

Bunch, T. J., Bair, T. L., Crandall, B. G., Cutler, M. J., Day, J. D., Graves, K. G., ... May, H. T. (2020). Stroke and dementia risk in patients with and without atrial fibrillation and carotid arterial disease. Heart Rhythm, 17(1), 20-26. https://doi.org/10.1016/j.hrthm.2019.07.007

Caruyer, E., Lenglet, C., Sapiro, G., \& Deriche, R. (2013). Design of multishell sampling schemes with uniform coverage in diffusion MRI. Magnetic Resonance in Medicine, 69(6), 1534-1540.

https://doi.org/10.1002/mrm.24736

Cavallari, M., Dai, W., Guttmann, C. R. G., Meier, D. S., Ngo, L. H., Hshieh, T. T., ... Alsop, D. C. (2017). Longitudinal diffusion changes following postoperative delirium in older people without dementia. Neurology, 89(10), 1020-1027. https://doi.org/10.1212/WNL.0000000000004329

De Rango, P., Caso, V., Leys, D., Paciaroni, M., Lenti, M., \& Cao, P. (2008, November 1). The role of carotid artery stenting and carotid endarterectomy in cognitive performance: A systematic review. Stroke, Vol. 39, pp. 3116-3127. https://doi.org/10.1161/STROKEAHA.108.518357

Dempsey, R. J., Jackson, D. C., Wilbrand, S. M., Mitchell, C. C., Berman, S. E., Johnson, S. C., ... Hermann, B. P. (2018). The Preservation of Cognition 1 Year After Carotid Endarterectomy in Patients With Prior Cognitive Decline. Neurosurgery, 82(3), 322-328. https://doi.org/10.1093/neuros/nyx173

Flaherty, M. L., Kissela, B., Khoury, J. C., Alwell, K., Moomaw, C. J., Woo, D., ... Kleindorfer, D. (2013). Carotid artery stenosis as a cause of stroke. Neuroepidemiology, 40(1), 36-41.

https://doi.org/10.1159/000341410 
Heller, S., \& Hines, G. (2017). Carotid Stenosis and Impaired Cognition. Cardiology in Review, 25(5), 211214. https://doi.org/10.1097/CRD.0000000000000139

Irfanoglu, M. O., Nayak, A., Jenkins, J., Hutchinson, E. B., Sadeghi, N., Thomas, C. P., \& Pierpaoli, C. (2016). DR-TAMAS: Diffeomorphic Registration for Tensor Accurate Alignment of Anatomical Structures. Neurolmage, 132, 439-454. https://doi.org/10.1016/j.neuroimage.2016.02.066

Jeurissen, B., Tournier, J. D., Dhollander, T., Connelly, A., \& Sijbers, J. (2014). Multi-tissue constrained spherical deconvolution for improved analysis of multi-shell diffusion MRI data. Neurolmage, 103, 411426. https://doi.org/10.1016/j.neuroimage.2014.07.061

Jones, D. K., Griffin, L. D., Alexander, D. C., Catani, M., Horsfield, M. A., Howard, R., \& Williams, S. C. R. (2002). Spatial normalization and averaging of diffusion tensor MRI data sets. Neurolmage, 17(2), 592617.

Kojima, D., Ogasawara, K., Kobayashi, M., Yoshida, K., Kubo, Y., Chida, K., ... Terasaki, K. (2016). Effects of uncomplicated carotid endarterectomy on cognitive function and brain perfusion in patients with unilateral asymptomatic severe stenosis of the internal carotid artery by comparison with unoperated patients. Neurological Research, 38(7), 580-586. https://doi.org/10.1080/01616412.2016.1188551

Lal, B. K., Dux, M. C., Sikdar, S., Goldstein, C., Khan, A. A., Yokemick, J., \& Zhao, L. (2017). Asymptomatic carotid stenosis is associated with cognitive impairment. Journal of Vascular Surgery, 66(4), 1083-1092. https://doi.org/10.1016/j.jvs.2017.04.038

Leemans, A., \& Jones, D. K. (2009). The B-matrix must be rotated when correcting for subject motion in DTI data. Magnetic Resonance in Medicine, 61(6), 1336-1349. https://doi.org/10.1002/mrm.21890

Lin, C.-J., Tu, P.-C., Chern, C.-M., Hsiao, F.-J., \& Chang, F.-C. (2014). Connectivity Features for Identifying Cognitive Impairment in Presymptomatic Carotid Stenosis. PLOS ONE, 9(1), 85441.

https://doi.org/10.1371/journal.pone.0085441

Manjón, J. V., Coupé, P., Concha, L., Buades, A., Collins, D. L., \& Robles, M. (2013). Diffusion Weighted Image Denoising Using Overcomplete Local PCA. PLoS ONE, 8(9), e73021.

https://doi.org/10.1371/journal.pone.0073021

Mito, R., Raffelt, D., Dhollander, T., Vaughan, D. N., Tournier, J. D., Salvado, O., ... Connelly, A. (2018). Fibrespecific white matter reductions in Alzheimer's disease and mild cognitive impairment. Brain, 141(3), 888-902. https://doi.org/10.1093/brain/awx355

Nakahara, M., Misaki, K., Tsutsui, T., Nakajima, R., Yoshikawa, A., Miyashita, K., ... Nakada, M. (2020). Effect of Unilateral Carotid Artery Stenting on Cognitive Function in Patients with Severe Bilateral Stenosis. World Neurosurgery, 135, 188-191. https://doi.org/10.1016/j.wneu.2019.12.068 
Porcu, M., Craboledda, D., Garofalo, P., Columbano, G., Barberini, L., Sanfilippo, R., ... Saba, L. (2019). Connectometry evaluation in patients undergoing carotid endarterectomy: an exploratory study. Brain Imaging and Behavior, 13(6), 1708-1718. https://doi.org/10.1007/s11682-018-0024-9

Power, M. C., Su, D., Wu, A., Reid, R. I., Jack, C. R., Knopman, D. S., ... Mosley, T. H. (2019). Association of white matter microstructural integrity with cognition and dementia. Neurobiology of Aging, 83, 63-72. https://doi.org/10.1016/j.neurobiolaging.2019.08.021

Raffelt, D., Tournier, J.-D., Fripp, J., Crozier, S., Connelly, A., \& Salvado, O. (2011). Symmetric diffeomorphic registration of fibre orientation distributions. Neurolmage, 56(3), 1171-1180.

https://doi.org/10.1016/j.neuroimage.2011.02.014

Ricotta, J. J., Aburahma, A., Ascher, E., Eskandari, M., Faries, P., \& Lal, B. K. (2011). Updated Society for Vascular Surgery guidelines for management of extracranial carotid disease: Executive summary. Journal of Vascular Surgery, 54(3), 832-836. https://doi.org/10.1016/j.jvs.2011.07.004

Rothwell, P. M., \& Goldstein, L. B. (2004). Carotid endarterectomy for asymptomatic carotid stenosis: asymptomatic carotid surgery trial. Stroke, 35(10), 2425-2427.

https://doi.org/10.1161/01.STR.0000141706.50170.a7

Smith, S. M., Jenkinson, M., Johansen-Berg, H., Rueckert, D., Nichols, T. E., Mackay, C. E., ... Behrens, T. E. J. (2006). Tract-based spatial statistics: Voxelwise analysis of multi-subject diffusion data. https://doi.org/10.1016/j.neuroimage.2006.02.024

Spencer, R. J., Wendell, C. R., Giggey, P. P., Katzel, L. I., Lefkowitz, D. M., Siegel, E. L., \& Waldstein, S. R. (2013). Psychometric Limitations of the Mini-Mental State Examination Among Nondemented Older Adults: An Evaluation of Neurocognitive and Magnetic Resonance Imaging Correlates. Experimental Aging Research, 39(4), 382-397. https://doi.org/10.1080/0361073X.2013.808109

Suemoto, C. K., Nitrini, R., Grinberg, L. T., Ferretti, R. E. L., Farfel, J. M., Leite, R. E. P., ... Pasqualucci, C. A. (2011). Atherosclerosis and dementia: A cross-sectional study with pathological analysis of the carotid arteries. Stroke, 42(12), 3614-3615. https://doi.org/10.1161/STROKEAHA.111.628156

Tournier, J.-D., Calamante, F., \& Connelly, A. (2007). Robust determination of the fibre orientation distribution in diffusion MRI: Non-negativity constrained super-resolved spherical deconvolution. Neurolmage, 35(4), 1459-1472. https://doi.org/10.1016/J.NEUROIMAGE.2007.02.016

Tournier, J.-D., Calamante, F., \& Connelly, A. (2012). MRtrix: Diffusion tractography in crossing fiber regions. International Journal of Imaging Systems and Technology, 22(1), 53-66.

https://doi.org/10.1002/ima.22005

Tustison, N. J., Avants, B. B., Cook, P. A., Yuanjie Zheng, Egan, A., Yushkevich, P. A., \& Gee, J. C. (2010). N4ITK: Improved N3 Bias Correction. IEEE Transactions on Medical Imaging, 29(6), 1310-1320. 
Veraart, J., Fieremans, E., Jelescu, I. O., Knoll, F., \& Novikov, D. S. (2016). Gibbs ringing in diffusion MRI. Magnetic Resonance in Medicine, 76(1), 301-314. https://doi.org/10.1002/mrm.25866

Wakana, S., Caprihan, A., Panzenboeck, M. M., Fallon, J. H., Perry, M., Gollub, R. L., ... Mori, S. (2007). Reproducibility of quantitative tractography methods applied to cerebral white matter. Neurolmage, 36(3), 630-644. https://doi.org/10.1016/j.neuroimage.2007.02.049

Wendell, C. R., Zonderman, A. B., Metter, E. J., Najjar, S. S., \& Waldstein, S. R. (2009). Carotid intimal medial thickness predicts cognitive decline among adults without clinical vascular disease. Stroke; a Journal of Cerebral Circulation, 40(10), 3180-3185. https://doi.org/10.1161/STROKEAHA.109.557280

Zhang, Y., Schuff, N., Du, A.-T., Rosen, H. J., Kramer, J. H., Gorno-Tempini, M. L., ... Weiner, M. W. (2009). White matter damage in frontotemporal dementia and Alzheimer's disease measured by diffusion MRI. Brain, 132(9), 2579-2592. https://doi.org/10.1093/brain/awp071

\section{Figures}

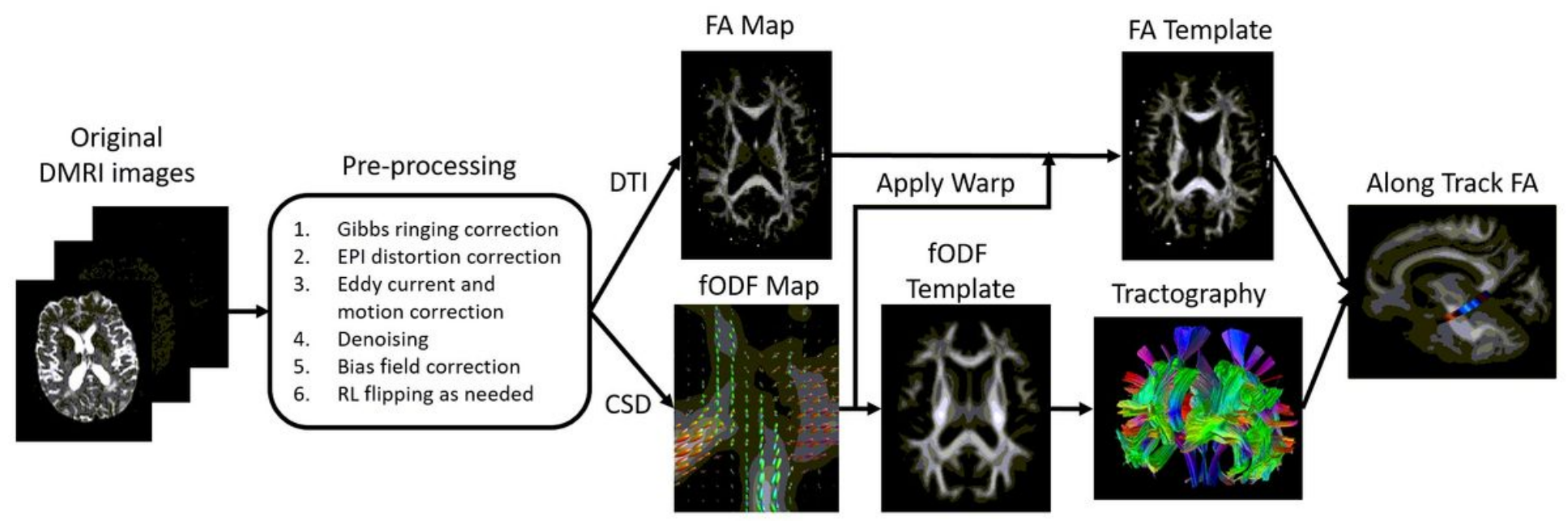

Figure 1

Graphical depiction of the diffusion MRI data processing pipeline. Original diffusion weighted images are first preprocessed to remove imaging artifacts including Gibbs ringing, EPI distortions, eddy current and motion distortion, noise, and image intensity bias from nonuniform coil sensitivities. If needed, images are flipped left to right to put the affected side on the left. The corrected data is fitted using both diffusion tensor imaging (DTI) to produce fractional anisotropy (FA) maps and constrained spherical deconvolution (CSD) to produce fiber orientation distribution functions (fODFs). The estimated fODFs are used to generate a study-specific fODF template. The warping used to register the subject fODFs to the study-specific fODF template is applied to individual subject FA maps to put the FA map into the same 

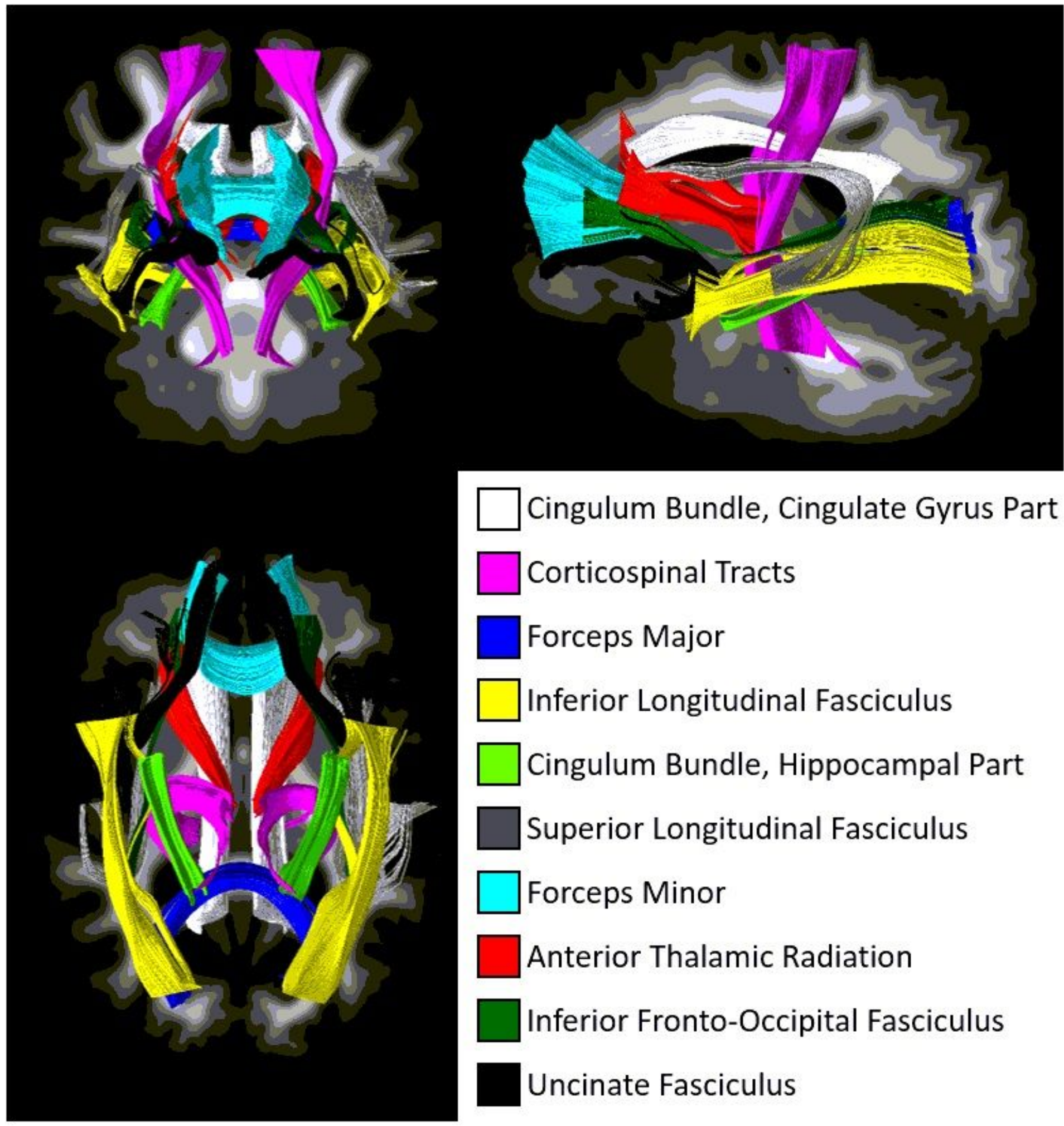

Cingulum Bundle, Cingulate Gyrus Part

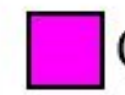

Corticospinal Tracts

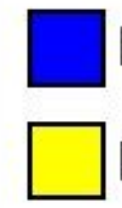

Forceps Major

$\square$ Inferior Longitudinal Fasciculus

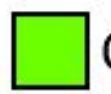

Cingulum Bundle, Hippocampal Part

Superior Longitudinal Fasciculus

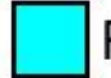

Forceps Minor

$\square$

Anterior Thalamic Radiation

Inferior Fronto-Occipital Fasciculus

Uncinate Fasciculus

\section{Figure 2}

Identification of the 18 major white matter pathways delineated using CSD-based probabilistic tractography and used for the analysis of along-track FA. Eight bilateral pathways and two interhemispheric pathways were analyzed. 

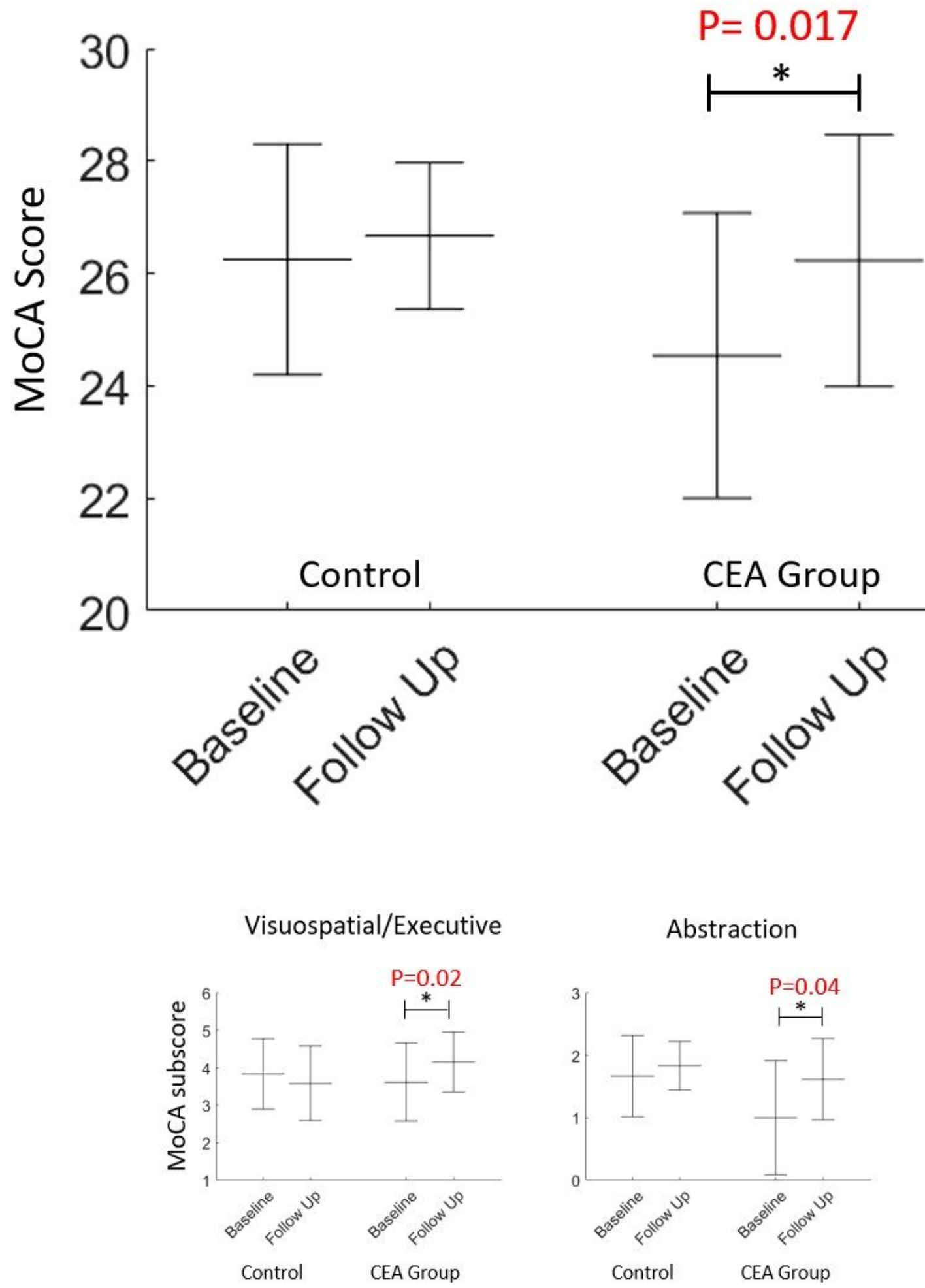
primarily by changes in the two MoCA cognitive subdomains (visuospatial/executive $(P=.02)$ and abstraction $(\mathrm{P}=.04)$ plotted below the MoCA scores.
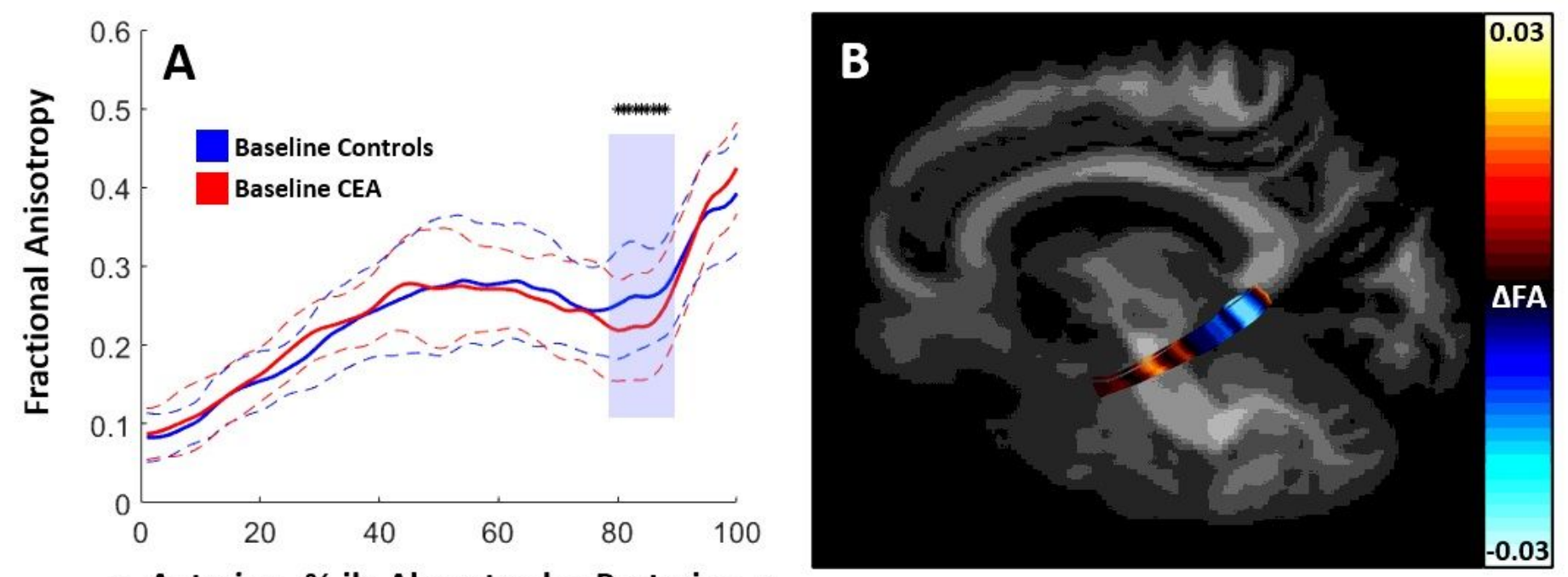

\section{<- Anterior - \%-ile Along track - Posterior ->}

\section{Figure 4}

A) Group averaged fractional anisotropy (FA) plotted along the hippocampal portion of the cingulum bundle at baseline for CEA (red) and control (blue) groups. Standard deviations are plotted as dashed lines above and below the average FA line. The average FA along the posterior portion of the hippocampus is significantly lower $(P<0.05)$ (denoted by * and highlighted area) in subjects with severe carotid artery stenosis. B) Differences in average FA along the hippocampus plotted on the CSD-based tractogram used to define the hippocampal part of the cingulum. Streamlines are blue where the average FA of the control group was lower than the CEA group, and red when the average FA was higher. 

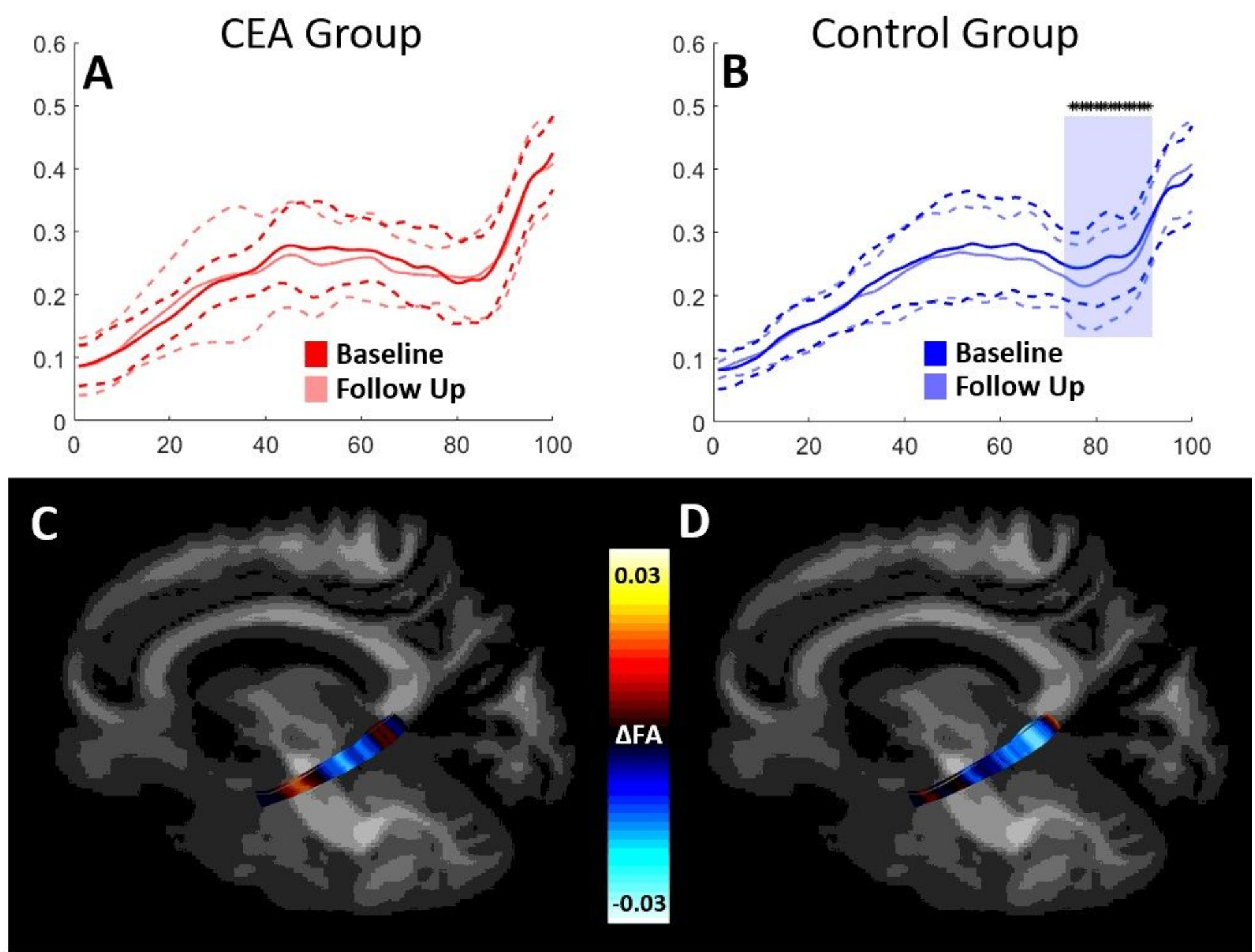

Figure 5

A) Group averaged fractional anisotropy (FA) plotted along the hippocampal portion of the cingulum bundle at baseline (blue) and follow-up (light blue) for control subjects. B) Group averaged fractional anisotropy (FA) plotted along the hippocampal portion of the cingulum bundle at baseline (red) and follow-up (light read) for the CEA group. Statistically significant decreases $(P<0.05)$ in FA (denoted by * and highlighted area in panel B) along the posterior portion of the hippocampus are not seen in the subjects who underwent CEA. Panels $C$ and $D$ demonstrate the average differences in FA in the subjects who underwent CEA (C), and in those that did not (D), plotted along the CSD-based tractograms used to define the hippocampal portion of the cingulum bundle. In panels $C$ and $D$, the streamlines are blue when the average FA in that region was lower at follow-up, and they are red when the average FA in that region was higher at follow-up.

\section{Supplementary Files}


This is a list of supplementary files associated with this preprint. Click to download.

- BIBBerman.docx

- BIBLeon.pdf

- brainimaging.pdf 